\title{
Solar Relativistic Proton Fluxes in the Solar Flare of 14 July 2000
}

\author{
Ruiguang Wang ${ }^{1,2}$ and Jingxiu Wang ${ }^{1}$ \\ ${ }^{1}$ National Astronomical Observatories, Chinese Academy of Sciences, Beijing 100012, China \\ ${ }^{2}$ Institute of Science and Technology for Opto-Electron Information, Yantai University, Yantai \\ 264005, China
}

\begin{abstract}
We studied the solar proton differential energy spectra with energy range of $1 \sim 500$ $\mathrm{MeV}$ at several time intervals during the 2000 July 14 solar flare. The results showed that before flare the spectra could be described by a power law function and after flare the power law spectra still existed above $30 \mathrm{MeV}$ although spectra became softer with time. There was a spectral "knee" occurring at $\sim 30 \mathrm{MeV}$. We constructed a solar proton differential spectrum from $30 \mathrm{MeV}$ to $3 \mathrm{GeV}$ at peak flux time 10:30 UT and fitted it in the same manner. On the basis of a supposition of having the same power law spectrum in higher energy, we calculated the solar proton integrated fluxes in energy range of from $500 \mathrm{MeV}$ to $20 \mathrm{GeV}$ and compared them with other results obtained from experimental, modeling and theoretical calculations in other big historic SEP events.
\end{abstract}

Keywords. Sun: coronal mass ejections (CMEs), particle emission

\section{Introduction}

Bastille Day event is one of the most important solar energetic events in this solar cycle. A X5.7/3B flare at position N22W07 in active region NOAA 9077 was well observed with ground- and space-based instruments. X-ray flare lasted from 10:03 to 10:43 UT with a peak at 10:24 UT. SOHO/LASCO observed a full halo earth directed CME with great speed at 10:54 UT and GOES-8 observed a rapid increase of energetic proton fluxes later. On ground, more than 20 neutron monitors (NMs) observed cosmic ray intensity increases ranging from $2 \%$ to $60 \%$. The earliest onset time of NMs increases was 10:30 UT and the highest rigidity was $6.7 \mathrm{GV}$. Modeling results from Vashenyuk et al. (2003) and Duldig et al. (2003) showed that the spectrum was soft with a power law index of between -5 and -7 during the rising phase for solar proton beam approaching the Earth. From the pitch angle distribution, it is seen that the particle arrival was anisotropic and also changed with time.

\section{Energy spectra}

Using GOES8 data, we constructed solar proton energy spectra at Earth in figure 1 for selected time intervals during July 14-16. The high energy range over $30 \mathrm{MeV}$ of the spectra were fitted by a power law function $d J_{E} / d E=A \times E^{-\delta}$. Spectral indices are showed in figure 2. Involving the flux calculated by Belov et al. (2001) from NM observations, the spectrum at peak time 10:30 UT was extended to $3 \mathrm{GeV}$ and fitted by power law function in energy range from $200 \mathrm{MeV}$ to $3 \mathrm{GeV}$, as shown in figure 3. Solar integrated spectra were plotted in figure 4, including spectra of other big historic GLE 


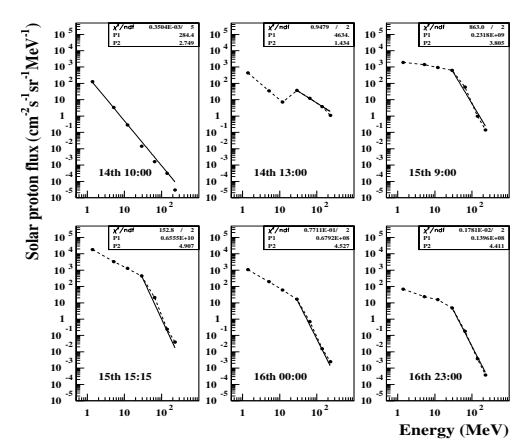

Figure 1. Solar proton energy spectra at Earth for selected time intervals during the GLE of 14 July 2000.

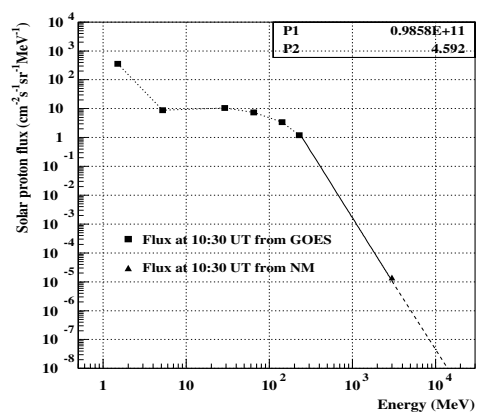

Figure 3. Solar proton energy spectrum at peak time 10:30 UT

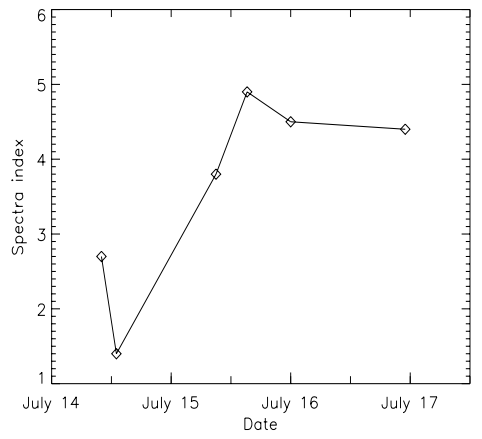

Figure 2. Spectral indices at selected time intervals during the GLE of 14 July 2000

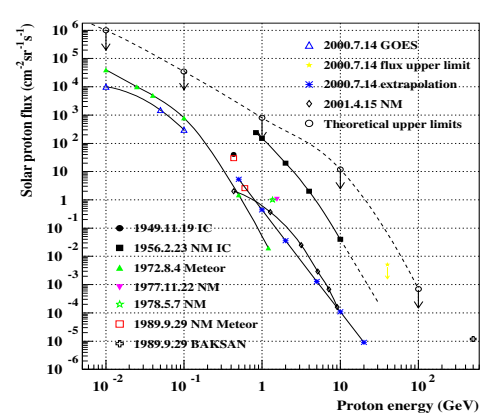

Figure 4. Integrated fluxes of some historic big GLE events.

events which were from theoretical and experimental calculations (see Miroshnichenko, 2001).

\section{Results}

For all time intervals shown in figure 1, a good fit could be obtained by using a power law in kinetic energy from $\sim 30 \mathrm{MeV}$ to $\sim 200 \mathrm{MeV}$, which was consistent with the diffusive coronal shock acceleration mechanism. The spectral index of $\sim-2.7$ before flare suggested particle behavior of galactic cosmic ray during solar quite. It is evident that the spectrum varies with time in both amplitude and shape and there is a spectral "knee" occurring at $\sim 30 \mathrm{MeV}$. Since relativistic protons peaked in intensity earlier than the lower energy protons, the spectrum was hard at the rising phase and became soft in the declining phase. From figure 4 it is seen that our estimated fluxes were comparable to that of other big GLE events.

\section{Acknowledgements}

The work is supported by the National Natural Science Foundation of China (10233050) and the National Key Basic Science Foundation (TG2000078404). 


\section{References}

Belov, A.V. et al. 2001, Proc. 27th ICRC, Hamburg, vol. 8, 3446.

Duldig, M.L., Bombardieri, D.J. and Humble, J.E. 2003, Proc. 28th ICRC, SH1.4, 3389.

Miroshnichenko, L.I. 2001, Solar cosmic rays, Kluwer Academic Publishers

Vashenyuk, E.V. et al. Proc. 28th ICRC, Tsukuba, SH1.4, 3401. 